\title{
Multiple infections of Anaplasma platys variants in Philippine dogs
}

\author{
Adrian Patalinghug Ybañez ${ }^{1,2,3}$, Rochelle Haidee Daclan Ybañez ${ }^{1}$, Naoaki Yokoyama ${ }^{4}$ and Hisashi Inokuma ${ }^{3}$
}

1. Department of Biology and Environmental Studies, College of Sciences, University of the Philippines Cebu, Lahug, Cebu City 6000, Philippines; 2. Department of Research, Gullas College of Medicine, University of the Visayas, Banilad, Mandaue City 6014, Cebu, Philippines; 3. Department of Veterinary Clinical Science, Obihiro University of Agriculture and Veterinary Medicine, Obihiro, Inada Cho, Hokkaido 080-8555 Japan; 4. Research Unit for Molecular Diagnosis, National Research Center for Protozoan Diseases, Obihiro University of Agriculture and Veterinary Medicine, Obihiro, Inada Cho, Hokkaido 080-8555, Japan.

Corresponding author: Adrian Patalinghug Ybañez, e-mail: dr.adrianpybanez@gmail.com, RHDY: rochelledybanez@gmail.com, NY: yokoyama@obihiro.ac.jp, HI: inokuma@obihiro.ac.jp Received: 25-07-2016, Accepted: 23-11-2016, Published online: 20-12-2016

doi: 10.14202/vetworld.2016.1456-1460 How to cite this article: Ybañez AP, Ybañez RHD, Yokoyama N, Inokuma H (2016) Multiple infections of Anaplasma platys variants in Philippine dogs, Veterinary World, 9(12): 1456-1460.

\begin{abstract}
Aim: Anaplasma platys, the causative agent of infectious canine cyclic thrombocytopenia, is a tick-borne pathogen that also has been implicated as potentially zoonotic. To provide molecular evidence on the multiple infections of $A$. platys variants in Philippine dogs.

Materials and Methods: DNA fragments of A. platys from infected dogs in the Philippines were molecularly characterized. For screening, 25 dogs suspected to have canine anaplasmosis were tested using a 16S rRNA-based nested polymerase chain reaction (PCR). Infection was confirmed by sequencing of positive amplicons. Second round PCR targeting a longer 16S rRNA fragment was subsequently performed on the first round PCR amplicons of the positive samples. Further characterization using the heat-shock operon ( $g r o E L)$ gene was also performed on the A. platys-positive samples.

Results: 10 16S rRNA sequences were obtained and found 99.6-100\% identical to each other and 99.6-99.7\% identical to the closest registered $A$. platys sequences. On the other hand, 36 groEL clone sequences were obtained and found to be $85.1-99.8 \%$ identical with each other and $85.0-88.9 \%$ identical to the closest previously registered $A$. platys sequences. Four dogs were found coinfected with 2-3 groEL variant sequences. Phylogenetic analyses suggest that the detected $A$. platys in the Philippines may represent unique variants.
\end{abstract}

Conclusion: A. platys variants were detected in Philippine dogs. Coinfection of different $A$. platys variants in dogs was also demonstrated. The present study may indicate the potential genetic diversity of $A$. platys in the country.

Keywords: Anaplasma platys, dogs, Philippines, variants.

\section{Introduction}

Anaplasma platys is a Gram-negative intracellular bacteria of the family Anaplasmataceae [1] that has recently been reported to infect humans [2-4]. In the Philippines, it has been detected in ticks [5] and dogs [6]. Nearby countries including Thailand [7], China [8], Korea [9], Japan [10], and Taiwan [11] already have reports of detection and characterization of this pathogen.

Characterizations of $A$. platys based on the heatshock operon gene ( $g r o E L)$ are limited. This particular gene has been used to identify genotypes of a related Anaplasma species. Pathogenicity of Anaplasma sp. maybe related to its genotype [12], although detailed investigations using groEL in assessing clinical signs or effects to the animal has been limited.

Only few reports on $A$. platys detection in the Philippines are available. The present study aimed to detect and molecularly characterize $A$. platys based on

Copyright: Ybañez, et al. Open Access. This article is distributed under the terms of the Creative Commons Attribution 4.0 International License (http://creativecommons.org/licenses/ by/4.0/), which permits unrestricted use, distribution, and reproduction in any medium, provided you give appropriate credit to the original author(s) and the source, provide a link to the Creative Commons license, and indicate if changes were made. The Creative Commons Public Domain Dedication waiver (http:// creativecommons.org/publicdomain/zero/1.0/) applies to the data made available in this article, unless otherwise stated. the 16S rRNA and groEL genes from infected dogs in the country. The obtained sequences were further subjected to phylogenetic analyses using different methods.

\section{Materials and Methods \\ Ethical approval}

The study was performed in accordance with the Institutional Animal Care and Use Committee guidelines of Southwestern University, Cebu and the Animal Welfare Act of the Philippines (RA8485), and with the approval of the attending veterinarians and proprietor of the veterinary establishment.

\section{DNA sample from dogs}

DNA samples were obtained from blood of 25 dogs that were presented at the GPY Veterinare Animale Group of Veterinary Clinics, Cebu, Philippines. These dogs were thrombocytopenic and exhibited clinical signs suggestive of canine anaplasmosis [13] and were found serologically negative for Ehrlichia canis using an Immunocomb ${ }^{\circledR}$ (Biogal, Israel) commercial test kit [14]. DNA was extracted using procedures as previously described [5].

\section{S rRNA polymerase chain reaction (PCR) assay}

For the 16S rRNA PCR, DNA samples were tested using a previously described method [5] with slight modification to confirm infection. Briefly, outer primer pair 
fD1/Rp2 and inner primer pair EHR16SD/EHR16SR (for screening) were used to amplify a final 345-bp-target of Ehrlichia/Anaplasma spp. Those found positive with the EHR16SD/EHR16SR screening PCR were further analyzed. Using the first round fD1/Rp2 amplicons, hemi-nested PCRs were performed utilizing the primer pairs fD1/EHR16SR and EHR16SD/Rp2 targeting a total of approximately $1400-1600 \mathrm{bp}$. A final volume of 10 and $25 \mu 1$ was set for the first and final round PCRs using a similar method, respectively [15]. The negative and positive controls used were double distilled water and $A$. bovis [16], respectively.

\section{groEL-based PCR assay}

DNA samples which were found positive during the screening PCR were further analyzed using a
groEL-based nested PCR assay. In the first round, primers EEgro1F and EEgro2R [17] were used employing procedures as previously described [18]. For the second round PCR, newly designed primers, i.e. APl136F (CCRGAGATKACKAAGGATGGC) and AP1590R (CCRCGRTCAAAY TGCATACC) that targeted a $455 \mathrm{bp}$ fragment were used. Similar final PCR volumes were used [18]. Step-down PCR method (with $2^{\circ} \mathrm{C}$ increment) was employed in the second round PCR with a starting and final annealing of $62^{\circ} \mathrm{C}$ and $52^{\circ} \mathrm{C}$, respectively.

\section{Cloning, sequencing, and sequence comparison}

Final amplicons were purified using a Gel Extraction Kit (QIAGEN, Valencia, CA, USA) and were cloned using a TA cloning kit (Invitrogen, USA).

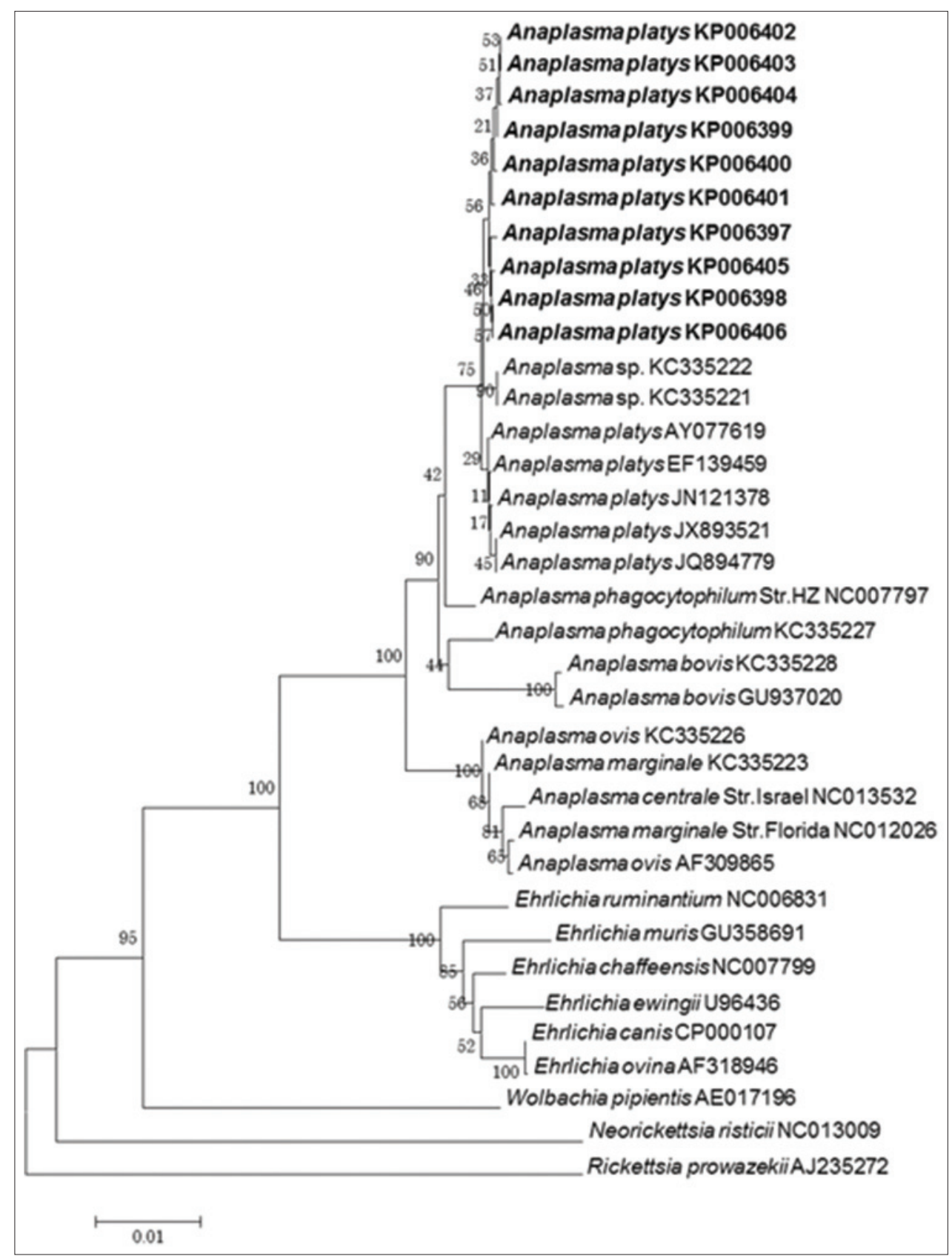

Figure-1: Phylogenetic analysis of Anaplasma platys detected from Philippine dogs based on the 16S rRNA gene using maximum likelihood method. Rickettsia prowazekii was set as the out-group. Sequences obtained in the study are set in bold. 
DNA sequencing was performed as described previously [18]. Obtained 16S rRNA and groEL sequences were aligned as previously described [18] to obtain partial but longer DNA fragments. Sequence comparison was also performed as previously described [18]. All sequences obtained in this study were registered at GenBank, USA.

\section{Phylogenetic analyses}

All sequences were manually trimmed to include only the sequence of interest. Percent identities were computed, and multiple sequence alignments were performed as previously described [18]. Analyses by neighbor-joining using maximum composite likelihood, by maximum likelihood with prior best model testing using MEGA 5 [19], and by Bayesian inference using MrBayes 3.2 [20] guided by the prior best model test results from MEGA 5 were employed. A total of 1000 bootstrap replications and 1,000,000 generations were used in the analyses using MEGA 5 and MrBayes 3.2, respectively. Tree results from MrBayes 3.2 were viewed using the FigTree v1.3.1 (http://tree. bio.ed.ac.uk/software/figtree/).

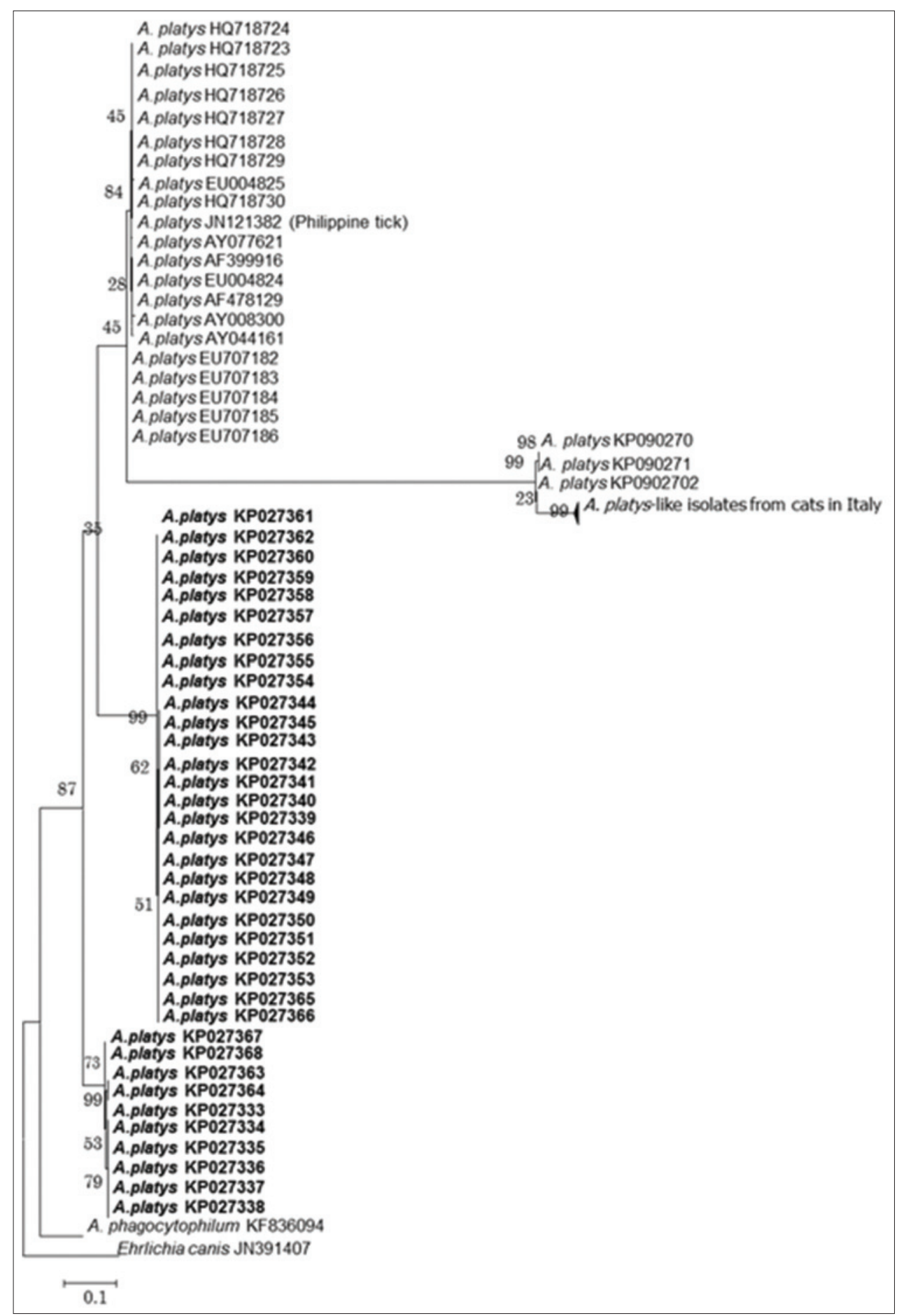

Figure-2: Phylogenetic analysis of Anaplasma platys detected from Philippine dogs based on the heat-shock operon ( $g r o E S L$ ) gene using maximum likelihood method (utilizing nucleotide sequences). Ehrlichia canis was set as the out-group. Sequences obtained in the study are set in bold. 


\section{Results and Discussion}

The screening PCR revealed 10 positive dogs. Obtained sequences (KP006398-KP006406) after alignment (1467 bp) were $99.6-100 \%$ identical to each other and $99.6-99.7 \%$ identical to the closest registered A. platys sequences from the Philippines and Thailand. From the A. platys-positive samples, further characterization using the groEL resulted to 36 clone sequences (KP027333-KP027368), which were 85.1$99.8 \%$ identical with each other and $85.0-88.9 \%$ identical to the closest registered $A$. platys sequences.

From the positive dogs, four were found coinfected with 2-3 groEL variant sequences. To the best of our knowledge, several studies have reported multiple infections of different tick-borne pathogens in dogs $[2,3,7,21]$, but multiple infections with different variants of a pathogen in an animal have not been reported until this study. While coinfections can produce more severe signs [7], further studies are needed to determine if coinfections with different variants of A. platys in dog have an effect on the clinical expression of the disease.

Regardless of the phylogenetic methods used, analyses based on the 16S rRNA gene showed that the obtained sequences formed a different $A$. platys subclade supported by moderate to high bootstrap values (Figure-1) or posterior probabilities (figure not shown). Interestingly, neutrophil-tropic Anaplasma spp. isolates detected from Mediterranean ruminants [22] clustered with the Philippine variants. Due to low intra-species variability of the 16S rRNA gene, analyses based on other genes such as groEL are needed to validate potential phylogenetic relationships.

Based on the groEL gene, phylogenetic analyses revealed that the detected Philippine A. platys variants formed a unique cluster (Figure-2). Owing to the higher number of newly obtained sequences in this study and higher variability of the gene, more subclades within the $A$. platys group were seen in the latter analyses. Moreover, new $A$. platys and $A$. platys-like isolates from Italy [23] were found to be in a unique subclade that appears to cluster differently from the detected Philippine $A$. platys variants. In this study, groEL was used to show the different variants of $A$. platys because of its higher intra- and inter-species variation than the $16 \mathrm{~S}$ rRNA $[13,18]$. Owing to this advantage, the groEL is used for genotyping other species [12,24]. Moreover, phylogenetic results using this gene have been shown to produce similar results using the ankA, gltA, and 16S rRNA [1,25-28].

Identities of the newly obtained sequences to registered $A$. platys sequences were confirmed by high similarities in two genes (16S rRNA and groEL). Phylogenetic analyses' results suggest that the detected A. platys in the Philippines may represent unique variants as the obtained sequences branched separately under the $A$. platys clade. These results can indicate the potential genetic diversity of $A$. platys in the country. This study supports the usefulness of groEL in investigating genetic diversities in Anaplasma species. On the other hand, further studies on the multiple infection of several A. platys variants in dogs that may also be coinfected with $E$. canis $[29,30]$, which has a common vector and is present in the Philippines.

\section{Conclusion}

A. platys variants were detected in Philippine dogs. Coinfection of different $A$. platys variants in dogs was also demonstrated. The present study may indicate the potential genetic diversity of A. platys in the country.

\section{Authors' Contributions}

APY and RHDY conceptualized the study and analyzed and wrote the manuscript. RRV contributed in the data analysis. NY and HI gave valuable insights and support in the conduct of the study.

\section{Acknowledgments}

The authors would like to thank Dr. Zandro O. Perez, Dr. Shirleny R. Gabotero, Dr. Reggie N. Fumar, Dr. Maxfrancis G. Talle, Mr. Cyrill John P. Godinez, and the staff of GPY Veterinare Animale, Cebu, Philippines, for their assistance, and the Ministry of Education, Culture, Sports, Science and Technology (MEXT) of Japan for the scholarship assistance.

\section{Competing Interests} interests.

The authors declare that they have no competing

\section{References}

1. Dumler, J.S., Barbet, A.F., Bekker, C.P.J., Dasch, G.A., Palmer, G.H., Ray, S.C., Rikihisa, Y. and Rurangirwa, F.R. (2001) Reorganization of genera in the families Rickettsiaceae and Anaplasmataceae in the order Rickettsiales: Unification of some species of Ehrlichia with Anaplasma, Cowdria with Ehrlichia and Ehrlichia with Neorickettsia, descriptions of six new species combinations and designation of Ehrlichia equi and 'HGE agent' as subjective synonyms of Ehrlichia phagocytophila. Int. J. Syst. Evol. Microbiol., 51: 2145-2165.

2. Maggi, R.G., Mascarelli, P.E., Havenga, L.N., Naidoo, V. and Breitschwerdt, E.B. (2013) Co-infection with Anaplasma platys, Bartonella henselae and Candidatus Mycoplasma haematoparvum in a veterinarian. Parasit. Vectors, 6: 103.

3. Breitschwerdt, E.B., Hegarty, B.C., Qurollo, B.A., Saito, T.B., Maggi, R.G., Blanton, L.S. and Bouyer, D.H. (2014) Intravascular persistence of Anaplasma platys, Ehrlichia chaffeensis, and Ehrlichia ewingii DNA in the blood of a dog and two family members. Parasit. Vectors, 1: 298.

4. Arraga-Alvarado, C.M., Qurollo, B.A., Parra, O.C., Berrueta, M.A., Hegarty, B.C. and Breitschwerdt, E.B. (2014) Molecular evidence of Anaplasma platys infection in two women from Venezuela. Am. J. Trop. Med. Hyg., 91: 1161-1165.

5. Ybañez, A.P., Perez, Z.O., Gabotero, S.R., Yandug, R.T., Matsumoto, K. and Inokuma, H. (2012) First molecular detection of Ehrlichia canis and Anaplasma platys in ticks from Dogs in Cebu, Philippines. Ticks Tick Borne Dis., 3: 288-293.

6. Ybanez, A.P. (2014) First molecular evidence of Ehrlichia canis infection in dogs with probable disease relapse in the 
Philippines. J. Adv. Vet. Res., 4: 184-188.

7. Suksawat, J., Xuejie, Y., Hancock, S.I., Hegarty, B.C., Nilkumhang, P. and Breitschwerdt, E.B. (2001) Serologic and molecular evidence of coinfection with multiple vector-borne pathogens in dogs from Thailand. J. Vet. Intern. Med., 15: 453-462.

8. Wen, B., Cao, W. and Pan, H. (2003) Ehrlichiae and Ehrlichial diseases in china. Ann. N. Y. Acad. Sci., 990: 45-53.

9. Kim, C.M., Yi, Y.H., Yu, D.H., Lee, M.J., Cho, M.R., Desai, A.R., Shringi, S., Klein, T.A., Kim, H.C., Song, J.W., Baek, L.J., Chong, S.T., O` Guinn, M.L., Lee, J.S., Lee, I.Y., Park, J.H., Foley, J. and Chae, J.S. (2006) Tick-borne Rickettsial pathogens in ticks and small mammals in Korea. Appl. Environ. Microbiol., 72: 5766-5776.

10. Inokuma, H., Beppu, T., Okuda, M., Shimada, Y. and Sakata, Y. (2003) Epidemiological survey of Anaplasma platys and Ehrlichia canis using ticks collected from Dogs in Japan. Vet. Parasitol., 115: 343-348.

11. Chang, A.C., Chang, W.L., Lin, C.T., Pan, M.J. and Lee, S.C. (1996) Canine infectious cyclic thrombocytopenia found in Taiwan. J. Vet. Med. Sci., 58: 473-476.

12. Haschke-Becher, E., Bernauer, R., Walleczek, A.M., Apfalter, P., Afazel-Saeedi, S., Kraus, J. and Strasser, P. (2010) First detection of the Anaplasma phagocytophilum groEL-A genotype in man. $J$. Infect., 60: 300-305.

13. Ybanez, A. (2013) First report on Anaplasma platys infection in a dog in the Philippines. Iran. J. Vet. Med., 7: 227-231.

14. Harrus, S., Alleman, A.R., Bark, H., Mahan, S.M. and Waner, T. (2002) Comparison of three enzyme-linked immunosorbent assays with the indirect immunofluorescent antibody test for the diagnosis of canine infection with Ehrlichia canis. Vet. Microbiol., 86: 361-368.

15. Ybañez, A.P., Sivakumar, T., Ybañez, R.H., Vincoy, M.R., Tingson, J.A., Perez, Z.O., Gabotero, S.R., Buchorno, LP., Inoue, N., Matsumoto, K., Inokuma, H. and Yokoyama, N. (2013) Molecular survey of bovine vector-borne pathogens in Cebu, Philippines. Vet. Parasitol., 196: 13-20.

16. Ybanez, A.P., Sashika, M. and Inokuma, H. (2014) The phylogenetic position of Anaplasma bovis and inferences on the phylogeny of the Genus Anaplasma. J. Vet. Med. Sci., 76(2): 307.

17. Chae, J.S., Foley, J.E., Dumler, J.S. and Madigan, J.E. (2000) Comparison of the nucleotide sequence of $16 \mathrm{~S}$ rRNA, 444 Ep-ank, and groESL heat shock operon genes in naturally occurring Ehrlichia equi and human granulocytic ehrlichiosis agent isolates from northern California. J. Clin. Microbiol., 38: 1364-1369.

18. Ybañez, A.P., Matsumoto, K., Kishimoto, T. and Inokuma, H. (2012) Molecular analyses of a potentially novel Anaplasma species closely related to Anaplasma phagocytophilum detected in sika deer (Cervus nippon yesoensis) in Japan. Vet. Microbiol., 157: 232-236.

19. Tamura, K., Peterson, D., Peterson, N., Stecher, G., Nei, M. and Kumar, S. (2011) MEGA5: Molecular evolutionary genetics analysis using maximum likelihood, evolutionary distance, and maximum parsimony methods. Mol. Biol. Evol., 28: 2731-2739.

20. Ronquist, F., Teslenko, M., van der Mark, P., Ayres, D.L.,
Darling, A., Höhna, S., Larget, B., Liu, L., Suchard, M.A. and Huelsenbeck, J.P. (2012) MrBayes 3.2: Efficient Bayesian phylogenetic inference and model choice across a large model space. Syst. Biol., 61: 539-542.

21. Gaunt, S., Beall, M., Stillman, B., Lorentzen, L., Diniz, P., Chandrashekar, R. and Breitschwerdt, E. (2010) Experimental infection and co-infection of dogs with Anaplasma platys and Ehrlichia canis: Hematologic, serologic and molecular findings. Parasit. Vectors, 3. DOI: 10.1186/1756-3305-3-33.

22. Zobba, R., Anfossi, A.G., Parpaglia, M.L.P., Dore, G.M., Chessa, B., Spezzigu, A., Rocca, S., Visco, S., Pittau, M. and Alberti, A. (2014) Molecular investigation and phylogeny of Anaplasma spp. in Mediterranean ruminants reveal the presence of neutrophil-tropic strains closely related to A. platys. Appl. Environ. Microbiol., 80: 271-280.

23. Zobba, R., Anfossi, A.G., Visco, S., Sotgiu, F., Dedola, C., Parpaglia, M.L.P., Battilani, M., Pittau, M. and Alberti, A. (2015) Cell tropism and molecular epidemiology of Anaplasma platys-like strains in cats. Ticks Tick Borne Dis., 6: 272-280.

24. Yushan, H., Lei, L., Weijia, L. and Xiaoguang, C. (2010) Sequence analysis of the groEL gene and its potential application in identification of pathogenic bacteria. Afr. $J$. Microbiol. Res., 4: 1733-1741.

25. Bown, K.J., Lambin, X., Ogden, N.H., Petrovec, M., Shaw, S.E., Woldehiwet, Z. and Birtles, R.J. (2007) Highresolution genetic fingerprinting of European strains of Anaplasma phagocytophilum by use of multilocus variable-number tandem-repeat analysis. J. Clin. Microbiol., 45: 1771-1776

26. Rar, V.A., Epikhina, T.I., Livanova, N.N., Panov, V.V., Doroschenko, E.K., Pukhovskaya, N.M. and Ivanov, L.I. (2011) Genetic variability of Anaplasma phagocytophilum in Ixodes persulcatus ticks and small mammals in the Asian part of Russia. Vector Borne Zoonotic Dis., 11: 1013-1021.

27. Yabsley, M.J., Murphy, S.M., Luttrell, M.P., Wilcox, B.R., Howerth, E.W. and Munderloh, U.G. (2008) Characterization of 'Candidatus Neoehrlichia lotoris' (family Anaplasmataceae) from raccoons (Procyon lotor). Int. J. Syst. Evol. Microbiol., 58: 2794-2798.

28. Inokuma, H., Fujii, K., Okuda, M., Onishi, T., Beaufils, J.P., Raoult, D. and Brouqui, P. (2002) Determination of the nucleotide sequences of heat shock operon ( $g r o E S L)$ and the citrate synthase gene ( $\mathrm{gltA}$ ) of Anaplasma (Ehrlichia) platys for phylogenetic and diagnostic studies. Clin. Diagn. Lab. Immunol., 9: 1132-1136.

29. Ybañez, A.P., Ybañez, R.H.D., Yokoyama, N. and Inokuma, H. (2015) RNA polymerase sub-unit $\beta$ (rpoB) characterization of Ehrlichia canis detected from dogs and Rhipicephalus sanguineus ticks in Cebu, Philippines. Philipp. Vet. Arch., 85: 601-608.

30. Ybañez, A.P., Ybañez, R.H.D., Villavelez, R.R., Malingin, H.P.F., Barrameda, D.N.M., Naquila, S.V. and Olimpos, S.M.B. (2016) Retrospective analyses of dogs found serologically positive for Ehrlichia canis in Cebu, Philippines from 2003 to 2014. Vet. World, 9: 43. 